\title{
PERFECT MAPS IN COMPACT (COUNTABLY COMPACT) SPACES
}

\author{
G.L. GARG and ASHA GOEL \\ Department of Mathematics \\ Punjabi University \\ Patiala-147 002, India
}

(Received January 23, 1993 and in revised form May 23, 1993)

AESTRACT. In this paper, among other results, characterizations of perfect maps in compact Hausdorff(Fréchet, countably compact, Hausdorff) spaces are obtained

KEY WORDS AND PHRASES. Perfect,compact, countably compact,continuous, countably compact preserving, compact preserving,closed, compact fibers,Fréchet space,k- space,closed graph.

1992 AMS SUBJECT CLASSIFICATION CODES. 54C05, 54C10, 54C25, 54D30

\section{INTRODUCTION.}

Throughout, by a space we shall mean a Hausdorff topological space. No map is assumed to be continuous or onto unless mentioned explicitly, $\mathrm{Cl}(\mathrm{A})$ will denote the closure of the subset $A$ in the space $X$. A map $f: X \rightarrow Y$ is said to be countably compact (compact[1] or proper in the terminology of Liden[2]) if inverse image of each countably compact (compact) set is countably compact (compact) $f$ is said to be countably compact preserving(compact preserving[3] or compact in the terminoloqy of Liden[2]) if image of each countably compact(compact) set is countably compact(compact). f is said to be perfect [2] if it is continuous, closed, and has compact fibers $f^{\prime}(y), y \in Y . X$ is said to be a Fréchet space if for each subset $A$ of $X, X \in \operatorname{cl}(A)$ implies there exists a sequence $\left\{x_{n}\right\}$ in $A$ converging to $x X$ is said to be a $k$-space if $O$ is open (equivalently:closed) in $X$ whenever $O \cap K$ is open(closed) in $K$ for every compact subset $K$ of $X$ Every Fréchet space as well as every locally compact space is a $\mathrm{k}$ - space.

The study of perfect maps and their properties can be found in Liden[2], Vainstein[4], Mishenko[5]. Kljusin[6], Borges[7], Bourbaki[8], Fedorcuk[9], Burke[10], Mancuso[11], Gao[12], Salmon[13], Arkhangel'skii \& Ponmarev[14], Ormotsadge[15], Daniels[16], Matveev[17], Frolık[18], Lin[19], Mihai[20] and Dugundji[21], to cite only a few.

In the present paper, among other results, characterizations of perfect maps in compact(countably compact) spaces are obtained.Examples are given to show that the condition ' Fréchet' on the domain and range spaces in our theorem 33 cannot be replaced by the weaker condition of ' $k$-space'.

2. PROPOSITIONS.

PROPOSITION 2.1. Let $f X \rightarrow Y$ be countably compact preserving(in particular,continuous), where $X$ is courtably 
compact and $Y$ is a Fréchet space Then $f$ is closed.

PROOF. The proof is the same as that of Theorem 16.19 of Thron[22].

PROPOSITION 2.2. Let $f: X \rightarrow Y$ be closed with compact (countably compact) fibers, where $X, Y$ are arbitrary. Then $f$ is compact (countably compact).

PROOF. For the compact version, see Theorem 3 of Liden[2]. His proof is valid for the non surjective case too. The proof of the other part is similar.

PROPOSITION 2.3. Let $f: X \rightarrow Y$ be countably compact, where $X$ is a Fréchet space and $Y$ is countably compact. Then $f$ is continuous.

PROOF. Let $F$ be a closed subset of $Y$. Then $F$ is countably compact. Since $f$ is countably compact, $f^{-1}(F)$ is countably compact and so closed subset of $X$, by Theorem 3.6 of Dugundji[21]. Hence $f$ is continuous.

Combining propositions 2.2 and 2.3, we get the following:

PROPOSITION 2.4. Let $f: X \rightarrow Y$ be closed with countably compact fibers, where $X$ is a Fréchet space and $Y$ is countably compact. Then $f$ is continuous.

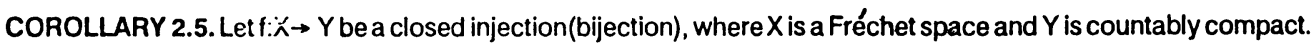
Then $f$ is an embedding(homeomorphism).

PROPOSITION 2.6. For any space $X$, let $f: X \rightarrow Y$ be compact, where $Y$ is a $k$-space. Then $f$ is closed.

PROOF. For proof, see Theorem 2 of Liden[2]. His proof is valid for the non surjective case too.

PROPOSITION 2.7. For any space $Y$, let $f: X \rightarrow Y$ be compact preserving with closed fibers, where $X$ is a $k$-space. Then $f$ is continuous.

PROOF. For proof, see Theorem 4 of Liden[2]. His proof is valid for the non surjective case too.

\section{THEOREMS.}

The following theorems 3.1 and 3.3 give characterizations of perfect maps in compact and countably compact spaces, respectively.

THEOREM 3.1. Let $f: X \rightarrow Y$ be any map, where $X, Y$ are compact spaces. Then the following are equivalent.

(a) $f$ is perfect.

(b) $f$ is continuous.

(c) $f$ is closed with closed fibers.

(d) f has closed graph.

(e) $f$ is compact.

(f) $f$ is compact preserving with closed fibers.

PROOF. Using proposition 2.2 above, and Theorem 1.1.10 of Hamlett and Herrington [23], the proof follows easily from the following diagram.

(a)
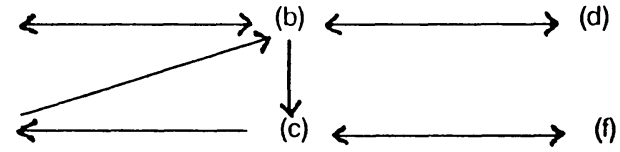

COROLLARY 3.2. Let $f: X \rightarrow Y$ be any injection (bijection), where $X, Y$ are compact spaces. Then the following are equivalent. 
(a) $f$ is an embedding(homeomorphism).

(b) $f$ is continuous.

(c) $f$ is closed(or open).

(d) f has closed graph.

(e) $f$ is compact.

(f) $f$ is compact preserving.

THEOREM 3.3. Let $f: X \rightarrow Y$ be any map with compact fibers, where $X, Y$ are Fréchet,countably compact spaces. Then the following are equivalent.
(a) $f$ is perfect.
(b) $f$ is continuous.
(c) $f$ is closed.
(d) f has closed graph.
(e) $f$ is compact.
(f) $f$ is compact preserving.
(g) $f$ is countably compact.
(h) $f$ is countably compact preserving.

PROOF. Using Theorem 5 of Piotrowski[24] and the above propositions 2.1 to 2.7, the proof follows easily from the following diagram.

(a)

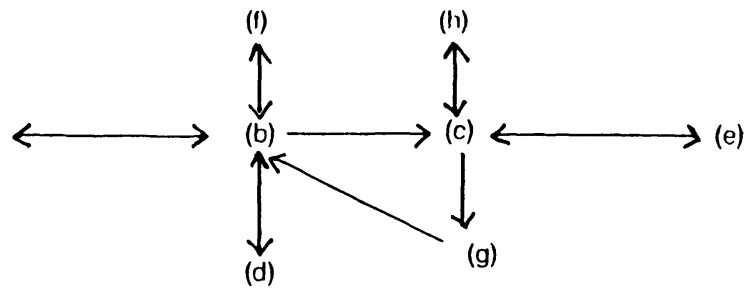

COROLLARY 3.4. Let $f: X \rightarrow Y$ be any injection(bijection), where $X, Y$ are Fréchet, countably compact spaces. Then the following are equivalent.
(a) $f$ is an embedding(homeomorphism).
(b) $f$ is continuous.
(c) $f$ is closed(or open).
(d) f has closed graph.
(e) $f$ is compact.
(f) $f$ is compact preserving.
(g) $f$ is countably compact.
(h) $f$ is countably compact preserving

NOTE 1.Theorem 3.3 and corollary 3.4 donot hold even for compact spaces $\mathrm{X}$ and $\mathrm{Y}$ which are non-Fréchet, as the following example shows.

EXAMPLE 3.5. Let $X=Y=[0, \Omega]$ be the ordinal space, where $\Omega$ is the first uncountable ordinal. Then the bijection 
$f Y \rightarrow Y$ which is iffentity on $(0, \Omega \Omega), f(\Omega)=0$, and $f(0)=\Omega$, is countably compact as well as countably compact preserving but does not satisfy any of the conditions (a) to $(f)$ of theoresn 33 and corollary 3.4 .

NOTE 2. Example 36 below shows that the condition 'Fréchet' on any of the spaces $X$ and $Y$ in theorem 3.3 cannot be replaced by ' $k$ - space'.

EXAMPLE 36 The inclusion map i $[0, \Omega) \rightarrow[0, \Omega]$ is continuous but not closed. Here the domain space is Fréchet, $T_{2}$, countably compact, and the range is compact, $T_{2}$. Also the function $f:[0, \Omega] \rightarrow[0, \Omega)$ which is identity on $[0, \Omega)$ and $f(\Omega)=0$, is closed but not continuous. Here the domain space is compact, $T_{2}$ and the range is Fréchet, $T_{2}$, and countably compact.

ACKNOWLEDGEMENT.The authors are grateful to the referee for useful suggestions.

\section{REFERENCES}

1. HALFAR,E. Compact mappings, Proc. Amer. Math Soc. $\underline{8}$ (1957), 828-830.

2. LIDEN,N. K-spaces, their anti-spaces, and related mappings,Pacific J. Math. 59(1975),505-514.

3.HALFAR, E. Condition implying continuity of functions, Proc. Amer. Math. Soc. 11(1960),688-691.

4. VAINSTEIN,I.A. On closed mappings of metric spaces,Dokl. Akad. Nauk SSSR (N.S). 57 (1947),319-321.

5 MISHENKO,A.S. Examples of irreducible perfect mappings of non normal spaces onto normal spaces, Uspekhi Mat. Nauk 18 (1963), 181-182.

6. KLJUSIN,V. Perfect mappings of paracompact spaces, Soviet Math. Dokl. $\underline{5}$ (1964), 1583-1586.

7. BORGES,C.J.R. On stratifiable spaces, Pacific J. Math. 17 (1966), 1-16.

8.BOURBAKI,N. General topology part-I.Addison- Wesley Publishing Company, 1966.

9 FEDORCUK, V.V. Irreducible perfect mappings and generalized proximities, Mat Sbornik.76 (1968),513-536.

10. BURKE, D.K. On subparacompact spaces, Proc.Amer. Math.Soc.23 (1969), 655-663.

11. MANCUSO,V.J.Inverse images and first countability, General Topology And Appl.2 (1972), 29-44.

12. GAO,G. Paracompactness and perfect mappings, Acta Math. Sinica. 23 (1980), 794-796.

13. SALMON, R.D. Perfect mappings and singular sets, Kyungpook Math. J. 21 (1981), 75-85.

14. ARKHANGEL'SKII,A.V. \& PONOMAREV.V.I. Fundamentals of general topology:Problems and Exercises, Hindustan Publishing Coroporaation(India) Delhi, 1984.

15. ORMOTSADGE,R.N Perfect mappings, Soobshch Akad Nauk Gruzin SSSR-119 (1985), 25-28.

16. DANIELS,P. Perfect pre-images of collectionwise normal spaces, Proc. Amer. Math. Soc. 97 (1986), 177-183.

17. MATVEEV,V.A. Perfect irreducible pre-images of topological spaces, Vestnik Moskov. Univ. Ser !. Mat. Mekh (1988),80-82.

18 FROLIK, Z Refinements of perfect maps onto metric spaces and an application to Cech-analytic spaces, Topology Appl 33 (1989), 77-84.

19. LIN,S Perfect pre-images of some generalized metrizable spaces, Questions Answers Gen. Topology $\underline{7}$ (1989), 23-30.

20 MIHAI,C. When closed maps are proper or continuous, Demonstratio Math. 22 (1989), 229-233.

21. DUGUNDJI,J Topology, Prentice-Hall of India Private Limited,New Delhi, 1975.

22.THRON,W J Topological structures, Holt,Rinehart and Winston, 1966.

23. HAMLETT,TR \& HERRINGTON, LL. The closed graph and p-closed graph properties in general topology, Amer Math Soc Providence Rhode Island, 1981.

24. PIOTROWSKI,Z \& SZYMANSKI,A Closed graph theorem topological approach, Rendiconti Del Circolo Matematico Di PalermoSerie II,37 (1988), 88-99. 


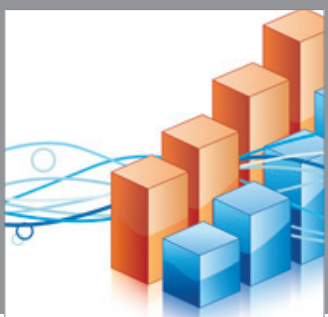

Advances in

Operations Research

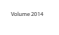

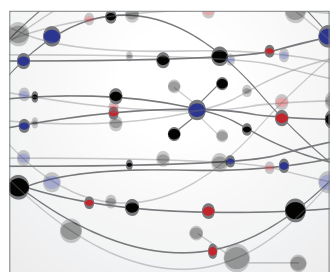

\section{The Scientific} World Journal
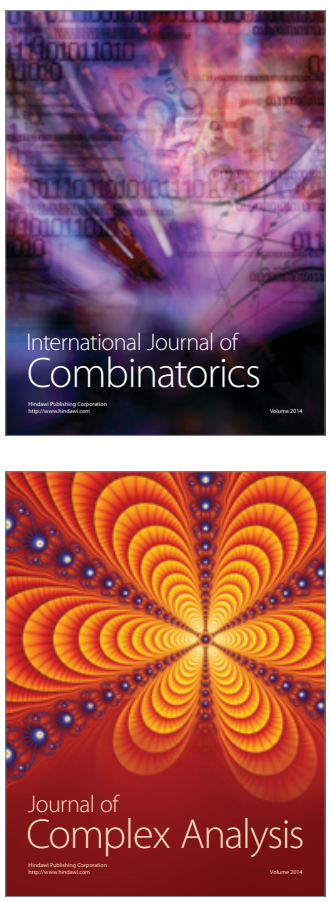

International Journal of

Mathematics and

Mathematical

Sciences
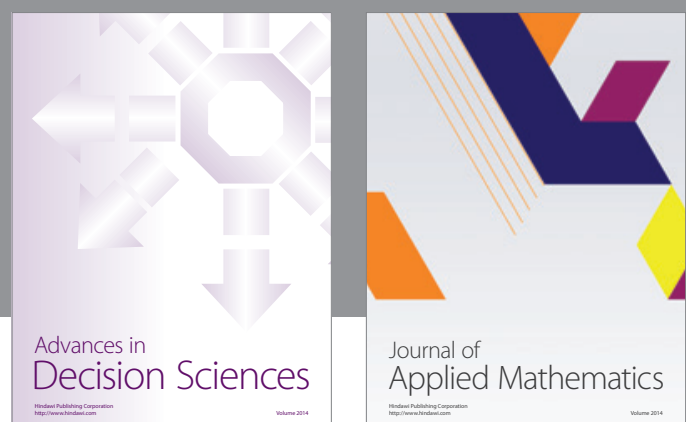

Journal of

Applied Mathematics
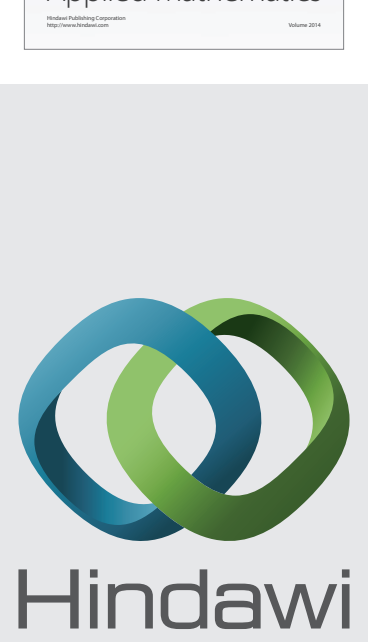

Submit your manuscripts at http://www.hindawi.com
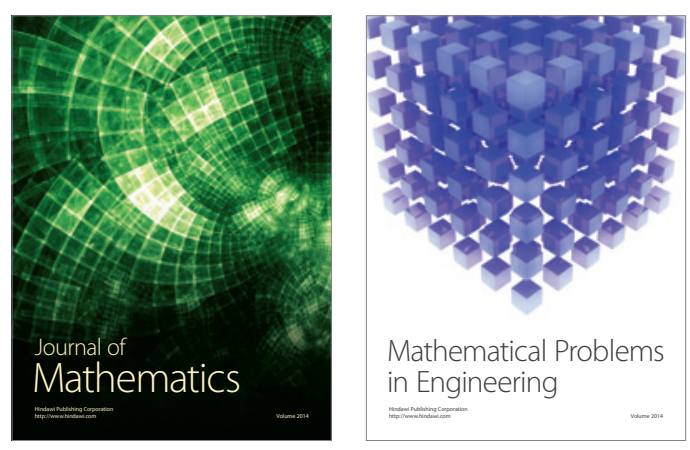

Mathematical Problems in Engineering
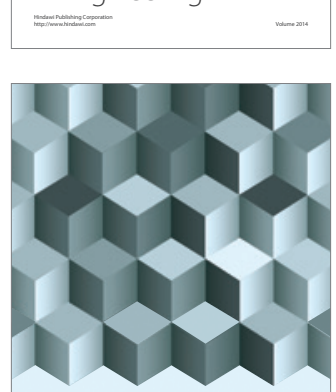

Journal of

Function Spaces
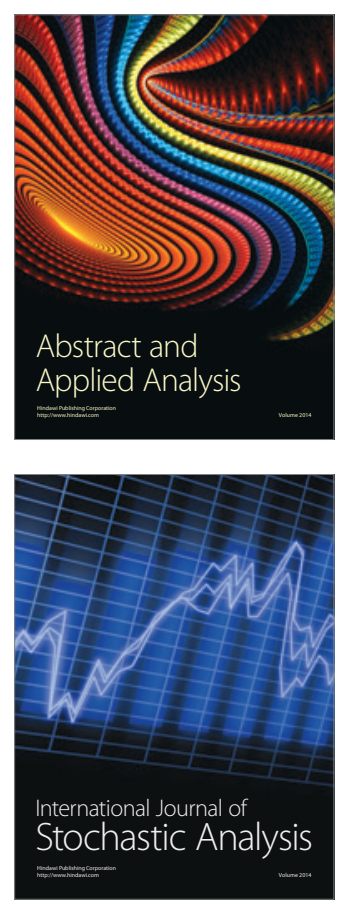

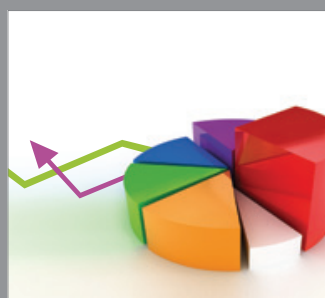

ournal of

Probability and Statistics

Promensencen
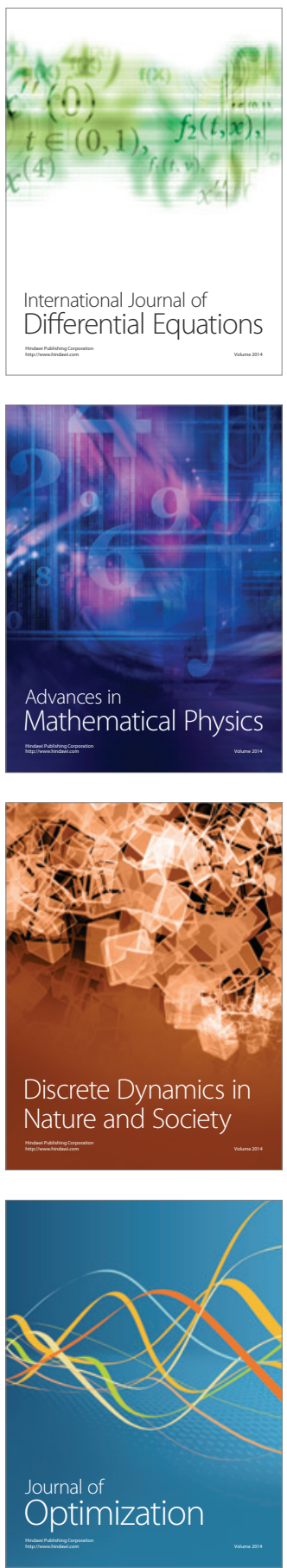